\title{
REGULACIÓN NEUROENDOCRINA DEL CICLO ESTRAL EN LA HEMBRA BOVINA
}

Melquisedec Ascanio Pérez. Médico Veterinario. Profesor Fisiología de la Reproducción Animal Universidad Francisco de Paula Santander

\section{RESÚMEN}

El conocimientos actual sobre la fisiología reproductiva ha modificado sustancialmente los conceptos que existían sobre la regulación del ciclo estral, situación que se ha producido básicamente a causa del desarrollo de la biotecnología de la reproducción, principalmente la transferencia de embriones y la fecundación in vitro, las que han exigido de un conocimiento cabal de los procesos de tipo estructural y funcional de los órganos que constituyen el eje hipotálamo-hipófisis-ovario, además se ha profundizado también en los roles protagónicos del útero y el embrión, en todo este complejo proceso.

El objetivo de esta revisión es exponer de modo accesible una visión general de la regulación neuroendocrina del ciclo estral, precisando los aspectos que constituyen los núcleos conceptuales de este proceso, de modo tal que pueda comprenderse, con relativa facilidad, las variaciones que frecuentemente se observan en el comportamiento del ciclo de las hembras bovinas.

Palabras claves:

Ciclo estral vaca, foliculogénesis, dinámica folicular, ovocitación.

\section{FOLICULOGÉNESIS}

La cantidad de folículos primordiales en los bovinos puede ser desde 130000 hasta 175000 según diferentes autores, en la porcina se reduce a 80000, mientras que en la humana la cifra es de 400000 .

La comparación entre especies de mamíferos sugiere que no existe relación aparente entre la magnitud de la población de folículos primordiales y el subsecuente tamaño de la camada o la duración de la vida reproductiva del adulto (Monniaux, et al, 1997). Los folículos primordiales inician su crecimiento y diferenciación en un proceso aparentemente continuo pero irreversible que es conocido como foliculogénesis. Cuando un folículo entra en el grupo de crecimiento, éste será conducido a uno de los dos hechos siguientes: la degeneración por atresia, proceso que afecta a la casi totalidad de los folículos o finalmente concluir en el proceso de ovulación (ovocitación), de modo que algunos investigadores han sugerido que en el caso particular de la vaca, por cada ovulación se eliminan más de 12 folículos a través de procesos de atresia (Blanco y Campo, 1997).

La foliculogénesis es el proceso por el cual una cantidad de folículos, constante en cada especie, y los ovocitos que estos contienen, maduran en cada ciclo estral; frecuentemente un solo folículo madura y concluye en la ovulación en la vaca durante cada ciclo estral, no obstante, ovulaciones dobles o múltiples pueden ocurrir de manera natural (Murphy y Pescador, 1997).

El proceso de desarrollo de los folículos es de forma ininterrumpida y su duración se ha calculado en unos 180 días. La maduración folicular se adelanta a la maduración del ovocito (ovogénesis) de modo tal que cuando el folículo concluye su desarrollo -folículo De Graaf- aún el ovocito se encuentra en un estadio de inmadurez (Monget and Monniaux, 1995).

El folículo primordial constituye la reserva ovárica y puede permanecer de modo indefinido en los ovarios, transformándose en folículo primario cuando es activado para su desarrollo, hasta este momento el folículo no es gonadotropo-dependiente, por tal razón todavía no se ha precisado la naturaleza del estímulo que produce la activación del folículo para su desarrollo ulterior (Blanco y Campo, 1997; Fortune, 1994).

Cuando la capa de células de la granulosa se transforma de aplanada a cuboidal y la teca interna comienza su diferenciación, al folículo en desarrollo se le denomina folículo primario. Su crecimiento hacia el siguiente estadio, que es el de folículo secundario, 
Regulación neuroendocrina del ciclo estral en la hembra bovina Melquisedec Ascanio Pérez

se completa por la proliferación de las células de la granulosa; los folículos en estos dos estadios se describen colectivamente como preantrales (Murphy y Pescador, 1997).

La ocurrencia del desarrollo durante la vida de los mamíferos y la relativa ausencia de atresia durante los estadios preantrales de la foliculogénesis sugiere que el control paracrino del desarrollo puede ser un proceso constitutivo en la granulosa, teca y células del estroma ovárico (Monget and Monniaux, 1995). El siguiente estadio de desarrollo se caracteriza por la formación de una cavidad en el folículo y la producción de líquido, estos son conocidos con el nombre genérico de folículos antrales. La tasa de crecimiento folicular es muy lenta en los folículos antrales pequeños o tempranos, necesitando más de dos semanas para alcanzar el diámetro de $0.3 \mathrm{~mm}$ (Lussier, et al, 1987). Sin embargo, el crecimiento de los folículos antrales grandes es rápido, su tasa más elevada parece ocurrir entre 1 y $2 \mathrm{~mm}$ de diámetro. Generalmente se acepta que la formación del antro folicular en los mamíferos es un evento influenciado por las gonadotropinas y que la FSH (hormona folículo estimulante) es la principal hormona responsable (Roche, 1996).

Durante toda esta etapa de transformación los folículos se encuentran bajo la influencia de las gonadotropinas FSH y LH (hormona luteinizante), señalándose de modo reiterado la importancia de la FSH en la formación del antro folicular, lo cual se ha podido reproducir por medios experimentales. En el folículo antral se han comenzado a diferenciar estructuras que cumplen una función importante en la liberación de los esteroides sexuales (estrógenos y andrógenos); estas estructuras conocidas clásicamente como membrana granulosa y teca interna tienen un comportamiento diferente a la acción gonadotrópica, es decir, la producción estrogénica $\left(E_{2}\right)$ es asumida solamente por las células de la granulosa bajo la influencia de la FSH sobre sus receptores desarrollados en la fase de folículo antral; sin embargo la teca interna, que es una estructura muy vascularizada, no tiene capacidad para producir
$E_{2}$ toda vez que en ella no se desarrollan receptores bioactivos para la FSH, mientras que sí tiene receptores para la LH, por tal razón, su producción esteroidea es la androgénica, la que es transformada en $\mathrm{E}_{2}$ a nivel de la granulosa folicular (Blanco y Campo, 1997).

Desde el punto de vista evolutivo, en el ovocito se han producido también cambios estructurales, pues en este se ha diferenciado una capa protectora de varias decenas de micras de grosor y constituida por una mucoproteina que envuelve al ovocito conocida como zona pelúcida. Existe, además, una protección de tipo histológico estructurada por células foliculares, provenientes, posiblemente, de la granulosa, conocida comúnmente como corona radiada.

Los folículos antrales tardíos, es decir, preovulatorios tienen otras producciones de carácter endocrino como las inhibinas y las activinas, las primeras son hormonas de la familia peptídica que pueden controlar la liberación de la FSH inhibiendo la respuesta a la GnRH. (Hormona liberadora de gonadotropinas)

\section{DINÁMICA FOLICULAR}

El uso de la ecografía ha confirmado el modelo teórico planteado por Rajakoski, quien propuso que los patrones de crecimiento y regresión folicular, tanto a nivel individual como en el total de la población, se desarrollarían durante todo el ciclo, pero no de forma continua sino muy relacionados en el momento o día de éste (Rajakoski, 1960).

El seguimiento ecográfico de los folículos durante todo el ciclo permite la descripción de los niveles de crecimiento y desarrollo foliculares. Así, el desarrollo folicular en la vaca se puede enmarcar en las etapas de reclutamiento, selección y dominancia (Savio, et al, 1987; Savio, et al, 1988; Sirois and Fortune, 1988). El reclutamiento folicular es el proceso por el que, bajo la responsabilidad de la FSH, los folículos antrales pequeños (2-3mm de diámetro) comienzan a crecer, iniciando así una onda de desarrollo; grupos, más 
que folículos aislados, son reclutados y este proceso se relaciona temporalmente con cambios medibles de la FSH circulante (Adams, et al, 1992). La ampliación antral es el medio preponderante del crecimiento del folículo durante este período. También hay evidencia de mitosis concomitante en las células de la granulosa complementarias, al menos en roedores (Oktay, et al, 1995).

Se han invocado factores intraováricos estimulados por la FSH, los que están involucrados en el proceso de reclutamiento folicular, los factores de crecimiento similar a la insulina (IGF-1) y las proteínas a las que éstos se ligan han sido implicados en la amplificación de la acción de la FSH. La IGF-1 potencializa el desarrollo del folículo, como un mitógeno, en los folículos pequeños (EI IGF-1 estimula el crecimiento de las células de la granulosa) y por su acción en conjunto con las gonadotropinas para inducir la esteroidogénesis en los folículos antrales (Monget and Monniaux, 1995; Oktay, et al, 1995; Monget, et al, 2002). Los folículos desarrollan la fase de desviación (comienzo de la diferenciación del crecimiento entre dos folículos grandes) y llegan a la dominancia en 4 días de la fase de selección partiendo de una cohorte de $+3 \mathrm{~mm}$ de diámetro, creciendo de un modo muy rápido (Ginther, et al, 1998).

Se ha podido observar que durante este período se produce un abrupto cambio en la concentración de FSH y en la pulsatilidad de la $\mathrm{LH}$; este cambio hormonal puede actuar como el estímulo para el inicio de la diferenciación de los futuros folículos dominantes y los subordinados; estos cambios hormonales apoyan la hipótesis que expresa la necesidad de una cantidad suficiente de $\mathrm{LH}$ y concentraciones mínimas de FSH para que se facilite la desviación folicular (Ginther, ef al, 1998b).

El proceso de selección se caracteriza por el bloqueo ejercido por el folículo más desarrollado sobre los restantes folículos de la misma cohorte de desarrollo, este efecto se produce a través de sustancias hormonales como las inhibinas y el estradiol, las que actúan disminuyendo la liberación de FSH, de modo que los niveles insuficientes de gonadotropinas afectan, esencialmente, el desarrollo de los folículos más pequeños; en esta fase existe un grupo reducido de folículos que escapan al proceso de atresia. Otro mecanismo que se invoca es el de los factores paracrinos como el de la producción del factor de crecimiento epidermal (FCE) que reduce la capacidad de los folículos pequeños para utilizar andrógenos (Blanco y Campo, 1997).

La dominancia se refiere al desarrollo de un folículo mientras los restantes sufren un proceso de atresia fisiológica. El folículo dominante es más sensible a la acción de las gonadotropinas que los restantes, por lo que a pesar de influir negativamente en la liberación de gonadotropinas $\mathrm{FSH}$, no sufre atresia, esto lo favorece también el IGF-1 (Blanco y Campo, 1997). Se ha señalado que el primer folículo dominante pertenece a una cohorte de folículos de $4-6 \mathrm{~mm}$ aparecidos el primer día del ciclo (definiéndose el día del celo como día 0 del ciclo) para alcanzar su máximo tamaño (13-16 mm) entre los días 7-8, pudiendo permanecer estable hasta el día 11, momento en que inicia su regresión la que concluye el día 16. El segundo folículo dominante puede ser ovulatorio o no, en dependencia de ciclos de 2 ó 3 ondas de maduración; si el ciclo es de solamente 2 ondas, el segundo folículo dominante aparece el día 11, mientras que si el ciclo se caracteriza por 3 ondas, entonces aparecerá el día 8 para hacerse evidente un tercer folículo dominante, en este caso ovulatorio, el día 16 del ciclo, (Fortune, et al, 1988; Knopf, et al, 1989; Roche and Boland, 1993) cuya tasa de crecimiento es mayor que la de los folículos de las primeras ondas (Kojima, et al, 2003).

Sin embargo, en un trabajo detallado y publicado por Sabio, et al, 1993, se observó la misma cantidad de vacas con 2 ó 3 ondas de desarrollo folicular. En las vacas con dos ondas, el folículo dominante fue identificado el día $2.6+0.7$ adquiriendo su máximo desarrollo durante el día 8.7 con un tamaño de 16.7 
Regulación neuroendocrina del ciclo estral en la hembra bovina Melquisedec Ascanio Pérez

$+0.7 \mathrm{~mm}$. La segunda onda fue detectada el día 15.3 +1.8 y su diámetro máximo de $18.7+0.9 \mathrm{~mm}$ el día $22+1.1$. Cuando los animales presentaron 3 ondas entonces la primera se identificó a los $2.2+0.5$, la segunda a los $11.0+0.8$ y la tercera a los $17.0+$ 0.4 días alcanzándose los diámetros máximos a los $7.4+0.4 ; 15.0+0.9$ y $22.2+0.2$ días respectivamente. La duración de los intervalos interestrales no evidenciaron diferencias apreciables, contrariamente a lo que se ha encontrado en otros estudios.

Por otra parte, Denis y Gil, 1997, utilizaron un ecógrafo de tiempo Real Aloka SSD-500 para el estudio de las ondas foliculares en un grupo de 22 vacas, observaron que el $75 \%$ de los animales presentaron solamente dos ondas de desarrollo, en ellos la segunda onda se inició los días 15 y 16 del ciclo. Estos mismos investigadores aplicaron las técnicas de radioinmuno análisis (RIA) para determinar los niveles de progesterona en suero, constatando que las mayores concentraciones fueron los días 10, 12, 14 y 16 del ciclo con valores de $8.8 ; 10.15 ; 17.00$ y $9.35 \mathrm{nmol} / \mathrm{l}$ respectivamente; concentraciones similares se reportaron en vacas lecheras y búfalos de río (Campo, et al, 1984; Campo, et al, 1985).

El control endocrino del crecimiento y desarrollo de nuevos folículos es atribuido a la gonadotropina FSH, toda vez que en el vacuno se ha descrito un incremento en los niveles de esta hormona cuando se cauterizan los folículos dominantes, asimismo, la administración de líquido folicular estimula la aparición de una cohorte de folículos con diametros de 4-5 mm, lo que ocurre de 24 a 48 horas posteriores a la administración. La competencia entre los folículos por la FSH circulante es la responsable de la dominancia; cuando se elimina el folículo dominante, el subordinado más cercano al dominante ocupa su lugar (Kastelic, et al, 1990; Ko, ef al, 1991; Adams, et al, 1993; Sunderland, et al, 1994).

La FSH es indispensable para la secreción de estrógenos foliculares ya que estimula el crecimiento, la mitosis y la completa diferenciación de las células de la granulosa de los folículos preovulatorios grandes, para que adquieran receptores para la $\mathrm{LH}$ y desarrollen su máxima actividad aromatizante. Cerca del $90 \%$ del estradiol secretado por los ovarios se deriva de estos folículos estimulados por la FSH (Rivera, 1993).

La transformación de los folículos ováricos durante el ciclo estral en el ganado está regulada por las concentraciones de $\mathrm{P}_{4}$ (progesterona) en plasma actuando vía feedback negativo, lo que tiene un efecto en la secreción de LH. La baja frecuencia de los pulsos de LH en la fase luteal que la caracteriza no es suficiente para mantener el continuo crecimiento de los folículos dominantes, por esta situación se ha formulado la hipótesis de que la inadecuada secreción de andrógenos por la teca interna limita la subsecuente función de las células de la granulosa, necesaria para terminar el desarrollo y la función de los folículos dominantes, los cuales no pueden suprimir prolongadamente el crecimiento de otros folículos y por ello se inicia otra nueva onda de desarrollo folicular (Savio, et al, 1993).

El papel de la FSH en la selección y dominancia ha sido demostrado por la administración de FSH exógena durante dos días dentro del período en que se prevé la selección de un folículo, esta aplicación retrasa la esperada selección, alterando significativamente los perfiles de crecimiento del dominante y aumentando el crecimiento y retrasando la regresión del primero y segundo folículo subordinado (Adams, et al, 1993). En los últimos años se ha reportado que en los folículos dominantes y los subordinados de la primera onda de maduración están presentes proteínas que se combinan con la IGF (insulin-like growth factor), estas proteínas han sido designadas por números de modo tal que su presencia se identifica por los símbolos siguientes: IGF $\mathrm{BP}-2,3,4$ y 5 . Estos hallazgos apoyan la hipótesis que plantea que los folículos dominantes pueden emplear, de manera más eficiente, los niveles existentes de FSH debido al aumento de la disponibilidad de los IGF y que la atresia en los folículos subordinados es el resultado de una reducción inducida por el IGF BP 
Regulación neuroendocrina del ciclo estral en la hembra bovina Melquisedec Ascanio Pérez

sobre los IGF (De la Sota, et al, 1996; Monniaux, et al, 1997).

Las concentraciones de IGF-1 varían de acuerdo a la condición corporal y no afectan sustancialmente el tamaño folicular (Spicer, et al, 2002).

Actualmente se sabe que la atresia folicular es un proceso que causa la muerte y desaparición de más del $99 \%$ de los folículos que entran a la población de crecimiento. La mayoría de la degeneración folicular ocurre en el estadio antral temprano, antes del reclutamiento (Murphy y Pescador, 1997).

La atresia puede engendrar pérdida de la vascularidad de la teca, degeneración del ovocito y picnosis de la granulosa, en un proceso de todo o nada. El medio más común de la degeneración celular fisiológica es por apoptosis o muerte celular programada (Peluso, et al, 1995).

La apoptosis fue identificada como mecanismo de muerte celular programada en los folículos atrésicos. Como se indicó anteriormente, las gonadotropinas y los factores de crecimiento son elementos esenciales para el mantenimiento de la población folicular, entre sus efectos pleiotrópicos está la prevención de la apoptosis. El efecto de las gonadotropinas puede ser directo, toda vez que la apoptosis puede suprimirse in vitro por tratamiento con agentes que minimizan las acciones de las gonadotropinas (Tilly, et al, 1991; Jolly, et al, 1994; Flows, et al, 1995).

De modo resumido se puede plantear que la primera onda de maduración se aprecia durante la primera semana posterior al celo, donde existe un folículo dominante que produce un pico de estrógeno los días 5 y 6 del ciclo, estos picos son de una magnitud discreta y se han relacionado con la vitalidad de los embriones en esa época y con la migración e implantación del mismo.

La segunda oleada de maduración ocurre durante la segunda semana del ciclo (10-15 días) relacionándose también con una descarga de estrógenos la cual está asociada a la estimulación en la síntesis en el útero, de receptores bioactivos para la oxitocina, lo que en su momento desempeña un papel fundamental en la producción y liberación de $\mathrm{PGF}_{2}$ a. Desde el punto de vista clínico la coincidencia de un folículo dominante con un cuerpo lúteo activo permite establecer el pronóstico aproximado del próximo celo, y por ende, reconocer si existe ciclo ovárico o si por el contrario estamos frente a un caso de anestro (aciclia).

La tercera onda de maduración sólo se presenta en una proporción de animales y la misma ocurre desde el día 17 hasta el momento de la ovulación. Los folículos dominantes preovulatorios pueden localizarse en la segunda y tercera onda de maduración en dependencia del ritmo circadiano de cada hembra.

\section{OVOCITACIÓN}

A pesar de lo controvertido de los diferentes enfoques y tendencias se han observado determinados pasos o eventos fisiológicos que pueden explicar lo esencial de este complejo proceso, lo que es posible resumir del modo siguiente:

- Aumento de la vascularización de toda la pared folicular, excepto en el ápice del mismo, donde se produce una zona avascular, la que representa el lugar por donde se romperá el folículo.

- Disociación de las células de la granulosa, lo que causa un adelgazamiento notable del grosor de la pared folicular.

- Disociación también de las células que conforman el cumulus ophorus liberándose el ovocito del maciso celular ovígero.

- La vascularización folicular preovulatoria condiciona los cambios edemáticos en la teca externa folicular y con ello se afecta la cohesión celular de la misma. Participando además una fuerte acción enzimática (colagenasa, fundamentalmente) que destruye la elasticidad del folículo representada, principalmente, por la teca externa. 
Regulación neuroendocrina del ciclo estral en la hembra bovina Melquisedec Ascanio Pérez

- En el ápice del folículo, aparecen en las células epiteliares los lisosomas que con sus hidrolasas destruyen las células de la túnica albuginea y las de la teca folicular.

- La pared folicular se prolapsa cónicamente produciéndose determinados abombamientos, conocidos comúnmente como estigma de ovulación, lugar por donde se romperá la pared folicular.

Momentos antes de la ruptura folicular se aprecia una elevación en la concentración de las prostaglandinas $\mathrm{F}_{2}$ a y $\mathrm{E}_{2}$, participando en la contracción ovárica y folicular, a causa de la cual se produce la expulsión del ovocito. En este momento participan también las enzimas que destruyen la cohesión de las fibras colágenas (Holy, 1987).

Se ha postulado que la descarga de $\mathrm{GnRH}$ es necesaria para que se produzca la ovulación en todos los mamíferos (Karsch,et al, 1997).

La inhibina y la folistatina son dos proteínas no relacionadas estructuralmente, que inhiben la secreción de FSH. Las activinas, las inhibinas y la folistatina no se producen únicamente en la gónada sino también en la hipófisis y tienen un efecto mudulador en la producción de FSH (Knight, P. G., and C. Glister. 2001).

La inhibina es un miembro de la familia de los factores de transformación y de crecimiento tipo b. Su estructura es heterodinámica con sub-unidades alfa y beta, las cuales existen en dos magnitudes de peso molecular en el líquido folicular bovino y ovino. $\left(O^{\prime}\right.$ shea, et al, 1994). Las concentraciones de inhibina en el líquido folicular están reguladas por las gonadotropinas. (Findlay, 1993). La activina es el hemodímetro más importante de las subunidades beta inhibina, la que incrementa más que inhibe la secreción de FSH.(36) La folistatina es una proteína de enlace de alta capacidad con baja afinidad por la activina, siendo el único mecanismo conocido por el cual ésta interfiere con la secreción de FSH (Sugino, et al, 1993).
En un modelo experimental bien concebido se comprobó que los niveles de $\mathrm{P}_{4}$ altos durante el período estral bloquean la liberación de LH con lo cual la duración del estro se prolonga, deprimiéndose las manifestaciones de éste; asimismo se comprobó que la ovulación demoraba más que lo esperado, lo que influyó significativamente en la fertilidad, de modo que hembras (novillas) que presentaron los niveles de $\mathrm{P}_{4}$ suprabasales durante el estro, solamente se fecundaron en el orden del $46 \%$, mientras que las que presentaron niveles bajos de $\mathrm{P}_{4}(0.4-0.5 \mathrm{nmol} / \mathrm{l})$ durante el celo mostraron una fertilidad elevada, es decir, se gestó el 90\%. (Duchens, 1969).

\section{CUERPO LUTEO}

Esta estructura funcional se desarrolla a partir de la cavidad folicular después de la ovulación, su desarrollo es sumamente rápido, su producción de progesterona es máxima desde el día 10 hasta el 16 del ciclo estral, aunque su nivel es importante ya desde el cuarto. día del ciclo. Produce $\mathrm{P}_{4}$ como su producto primario, aunque secreta además prostaglandinas y una variedad de hormonas peptídicas y proteícas como la relaxina, oxitocina, vasopresina y estradiol en algunas especies (Niswender, et al, 1994).

El CL está constituido por las células de la teca interna (pequeñas y activas en la primera etapa de su desarrollo) y células de la granulosa (grandes y activas en la segunda mitad de su desarrollo). Según Rivera, 1993, las características principales de cada tipo celular puede resumirse de la forma siguiente:

Las células pequeñas son más sensibles a la acción de la LH pues poseen una cantidad mayor de receptores biológicamente activos para esta hormona y la respuesta es mediada por el sistema AMPc - adenilato ciclasa.

Las células luteales grandes tienen menos receptores para la LH (la mayor cantidad es para la $\mathrm{PGF}_{2}$ a y la $\mathrm{PGE}_{2}$ ) son las encargadas de la producción de oxitocina luteal. Contrariamente a las pequeñas, la 
Regulación neuroendocrina del ciclo estral en la hembra bovina Melquisedec Ascanio Pérez

producción de $\mathrm{P}_{4}$ está controlada, al menos parcialmente, por el sistema $\mathrm{Ca}^{+{ }^{+}}{ }_{\text {-Fosfatil inositol }}$ mositol-Quinasa c.

Ambos tipos de células secretan $\mathrm{P}_{4}$, pero las células pequeñas han mostrado poseer mayor cantidad de receptores para la $\mathrm{LH}$ y su respuesta secretora ante esta gonadotropina in vitro ha sido 6 veces mayor que en las células grandes. Las células pequeñas representan aproximadamente el $20 \%$ del total de la población de células luteales (Niswender, et al, 1994). Las células grandes presentan casi la totalidad de los receptores para las prostaglandinas $E_{2}$ y $F_{2}$ alfa. La alta concentración y afinidad por los receptores específicos para la $\mathrm{PGF}_{2}$ a en el bovino es similar los días 2, 4, 6 y 10 del ciclo estral lo que hace difícil explicar la falta de efectos de la PGF $_{2}$ a antes de los días 5 y 6 del ciclo estral (Wiltbank, et al, 1991).

La regulación de la función de esta estructura es un mecanismo complejo; en ella intervienen factores tróficos y líticos que están presentes en forma concurrente durante todo el ciclo estral y por tanto la estimulación o la inhibición de la síntesis y secreción de $\mathrm{P}_{4}$ depende del balance entre estos factores (Rivera, 1993). Un conjunto importante de investigaciones in vivo e in vitro han demostrado que la $\mathrm{LH}$ es el factor luteotrópico más importante en la vaca.

La lísis del CL se puede producir como consecuencia de la interacción hormonal sobre sus receptores, donde intervienen principalmente la $\mathrm{PGF}_{2} \mathrm{a}$, la oxitocina y los estrógenos. Se reconoce la intervención directa de la $\mathrm{PGF}_{2} \mathrm{a}$ en la lísis luteal, no obstante su síntesis y liberación está motivada por las concentraciones de otras hormonas como la oxitocina hipofisaria y luteal, estrógenos, incluso la $\mathrm{P}_{4}$ parece desempeñar un papel fundamental en este mecanismo (Blanco y Campo, 1997). En trabajos publicados por Silvia et al (Silvia and Taylor, 1989; Silvia, et al, 1991), se resume un modelo teórico coherente que ilustra esta compleja interacción hormonal.
En estos trabajos aparecen varios puntos en los que la $P_{4}$ y $E_{2}$ pudieran interactuar para promover la máxima secreción de $\mathrm{PGF}_{2}$ a durante la luteólisis. Primero las concentraciones de $\mathrm{E}_{2}$ durante la fase luteal (que como se sabe es baja) pudieran ser útiles para inducir un patrón apropiado de secreción de oxitocina neurohipofisiaria. Después de una exposición prolongada a la $\mathrm{P}_{4}$ el útero se vuelve sensible a la oxitocina y entonces la PG es secretada en respuesta a la oxitocina hipofisiaria; en estas condiciones la retroalimentación positiva está iniciada y el primer pulso de $\mathrm{PGF}_{2}$ a es liberado, comenzando así la luteólisis. Como los niveles de $\mathrm{P}_{4}$ bajan, la fase final de maduración folicular empieza y las concentraciones de estradiol circulante aumentan, en este momento la capacidad uterina para sintetizar y secretar PG alcanza sus niveles máximos a causa de la retirada de la $\mathrm{P}_{4}$ que desciende y/o incrementa el estradiol. Se describen dos fases diferentes de secreción de PG uterina a partir de la magnitud de sus pulsos, lo que se ha comprobado en la vaca y oveja. La secreción de PG se produce de un modo gradual en la magnitud de los pulsos durante la luteólisis; la posible dependencia al $\mathrm{E}_{2}$ en esta última fase de secreción pudiera, en parte, explicar lo esencial de los folículos para que se desarrolle el proceso de luteólisis. El $E_{2}$ pudiera contribuir a la regulación de la síntesis y secreción de la oxitocina hipofisiaria (neurohipofisiaria). En la oveja se ha comprobado que la administración de dosis bajas de $\mathrm{E}_{2}$ produce un patrón pulsátil de oxitocina neurohipofisiaria que es similar al observado en animales durante la fase folicular del ciclo estral. Un patrón similar se produce cuando se retira la $\mathrm{P}_{4}$ después de 10 días de influencia. Desde hace muchos años se conoce que la inyección de $E_{2}$ en animales sanos ovariectomizados inducen descargas de PG en 6-8 horas.

Es importante señalar que la $P_{4}$ estimula la acumulación de fosfolípidos triglicéridos en los tejidos endometriales, de modo tal que su acción es esencial para que se pueda sintetizar la PGF $_{2}$ a a partir del ácido araquidónico. Se ha comprobado además que la $\mathrm{P}_{4}$ inhibe la síntesis de receptores para el $\mathrm{E}_{2}$ y como 
Regulación neuroendocrina del ciclo estral en la hembra bovina Melquisedec Ascanio Pérez

es conocido la síntesis de los receptores de la oxitocina es un proceso estradiol-dependiente, lo que significa que si la $\mathrm{P}_{4}$ impide o limita la formación de receptores para el $E_{2}$ se produce entonces una incapacidad para la síntesis de los receptores para la oxitocina, limitándose así la síntesis y liberación de PG.

Actualmente se supone que el mecanismo disparador para la producción de PG es el estrógeno, que permite la acción de la oxitocina hipofisiaria en el útero, posteriormente la oxitocina luteal establece una retroalimentación positiva con la $\mathrm{PGF}_{2}$ a acelerándose la luteolisis en la fase final del CL. Sin embargo, este ciclo de retroalimentación se auto-limita debido a que los receptores para la oxitocina son destruidos después de ser utilizados, por lo que el útero deja de responder con liberación de $\mathrm{PGF}_{2}$ a.

Se ha confirmado que durante el ciclo estral normal, la duración de la fase lútea depende, esencialmente, del momento en que la frecuencia de la liberación de $\mathrm{PGF}_{2} \mathrm{a}$ llegue a un pulso rítmico de 5 a 6 horas (Zarco, 1995).

Se desarrolló un trabajo con vacas de carne mestizas con el objetivo de determinar la concentración de los receptores para la progesterona y la oxitocina en hembras que presentaban una fase luteal normal o corta, con la finalidad de encontrar una explicación científicamente fundamentada de la liberación precoz de la $\mathrm{PGF}_{2}$ a. Se concluyó que la concentración de receptores en el endometrio para la $\mathrm{P}_{4}$ el día 5 del ciclo fue mayor en las vacas con ciclo estral normal, mientras que aquellas que tuvieron un ciclo estral muy corto presentaron una concentración de receptores para la oxitocina mayor. Esto explica que en las hembras con ciclo corto el endometrio pierde la dominancia progesterónica, lo que posibilita la presencia abundante de receptores para la oxitocina y por ello una producción anticipada de $\mathrm{PGF}_{2}$ a y consecuentemente una destrucción precoz del CL y un ciclo estral muy corto (Zollers, et al, 1995).
El embrión es el responsable de indicar al útero su presencia para que éste responda suprimiendo el establecimiento del patrón de secreción pulsátil de $\mathrm{PGF}_{2} \mathrm{a}$; el embrión utiliza para ello un mensaje químico para lograr el reconocimiento materno de la gestación (Hunter, 1991). En el caso de los rumiantes la sustancia producida por el embrión para llevar este mensaje al endometrio es una proteína trofoblástica denominada Proteína Trofoblástica Bovina 1 (bTP-1) la cual se produce con mayor abundancia, después de los 15 días de fecundación, esta proteína se combina con los receptores existentes en el endometrio bloqueando la liberación pulsátil de $\mathrm{PGF}_{2}$ a. Es evidente que existe muy poco tiempo entre el momento en que el embrión puede señalar su presencia y el momento en que el útero materno está programado para destruir el cuerpo lúteo; esto explica que una causa importante de infertilidad en rumiantes es la regresión del $\mathrm{CL}$ antes que el embrión informe de su presencia, desarrollándose la asincronia materno embrionaria lo que se produce a causa de un retraso en el desarrollo embrionario o un adelanto en la liberación de $\mathrm{PGF}_{2}{ }^{a}$ por parte del útero materno.

En la práctica reproductiva actual, se ha utilizado la administración del interferón recombinante Bovino a 1 , para suplir esta demora en la producción de bTP-1, pues su similitud estructural así lo permite (Newton, et al, 1990; Roberts and Smith, 1992).

Se expone a continuación la representación esquemática de la regulación neuroendocrina del ciclo estral, destacándose aquellos aspectos más generales y básicos del mismo, requeridos para lograr una estructura conceptual coherente y sencilla en relación a este complejo y dinámico problema.

En la figura 1 se expone la representación esquemática de la regulación neuroendocrina del ciclo estral, destacándose aquellos aspectos más generales y básicos del mismo, que permite lograr una estructura conceptual coherente y sencilla en relación a este complejo y dinámico problema. 
Regulación neuroendocrina del ciclo estral en la hembra bovina

Melquisedec Ascanio Pérez

SISTEMA DE CONTROL Y REGULACION NEUROHORMONAL DEI. CICLO ESTRAL.

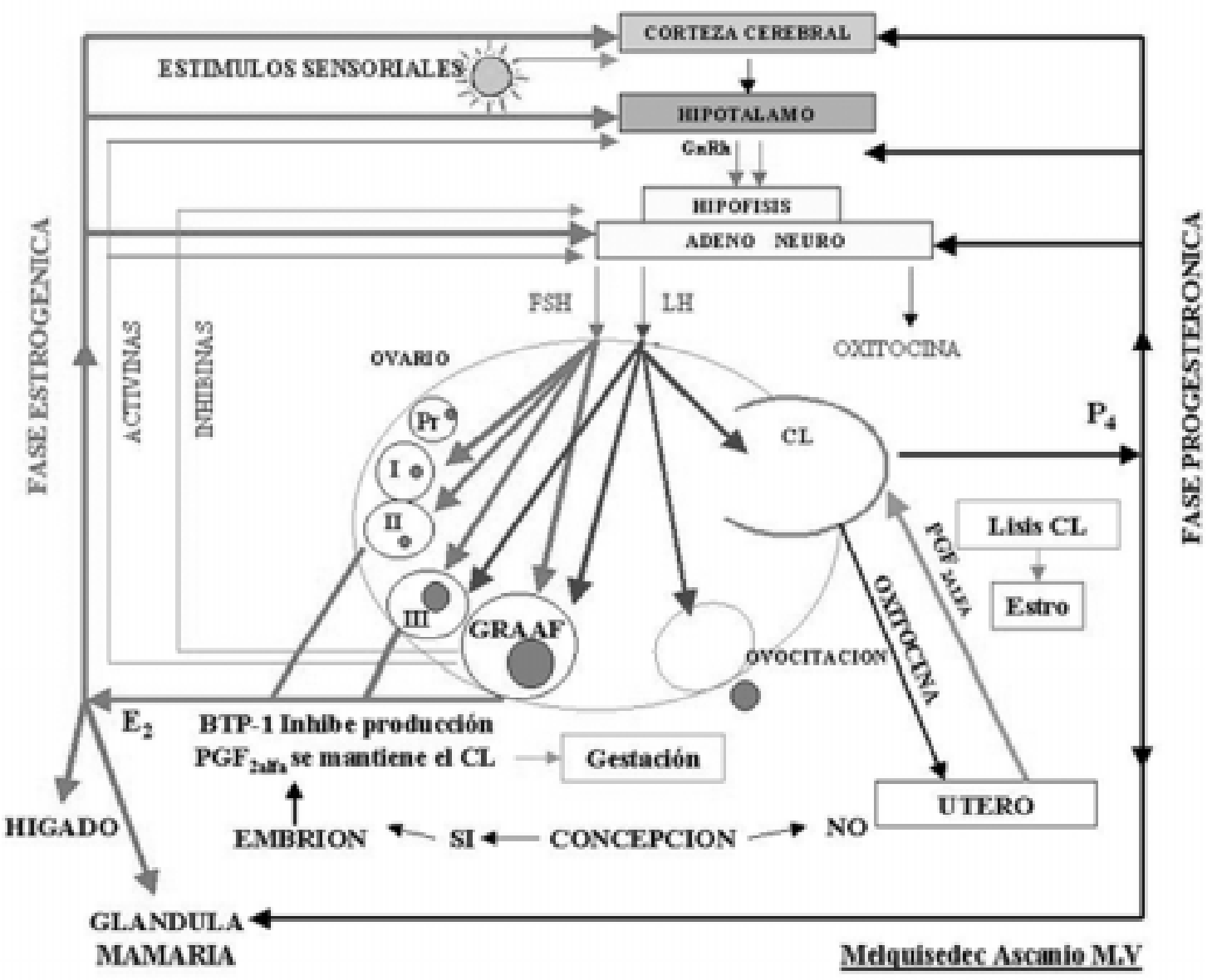

Figura 1

\section{BIBLIOGRAFÍA}

Adams, G.P.; Kot, K.; Smith, C.A. and Ginther, O.J. Selection of a dominant follicle and supression of follicular growth in heifers. Anim. Reprod. Sci. 30: 259271. 1993.

Adams, G.P.; Matteri, R.L.; Kastelic, J.P.; Ko, J.C.H. and Ginther, O.J. Association between surges of folliclestimulating hormone and the emergence of follicular waves in heifers. J. Reprod. Fert. 94: 177-188. 1992.

Blanco, A.G.S. y Campo, P.E. Mecanismos neurohormonales del ciclo estral en la vaca. Monografía. La Habana. 1997.
Campo, E.; Alonso, J.C. y García Libertad. Influencia de la época del año en el comportamiento reproductivo de búfalos. Rev. Salud Animal. 6: 27-29. 1984.

Campo, E.; Rizo, J.M. y García Libertad. Evaluación clínica del puerperio en vacas lecheras. Rev. Salud Animal. 7: 477-484. 1985.

De la Sota, R.L.; Simmen, F.A.; Diaz, T. and Thatcher, W.W. Insulin-like growth factor system in bovine firstwave dominant and subordinate follicle. Biol. Reprod. 55: 803-812. 1996.

Denis, G. y Gil, A. Aplicaciones prácticas de la ultrasonografía en los programas de transferencia de 
Regulación neuroendocrina del ciclo estral en la hembra bovina Melquisedec Ascanio Pérez

embriones. Centro de Investigación para la Mejora Animal. La Habana. Cuba. 1997.

Duchens, M. Influence of suprabasal progesterone on preovulatory follicle development in heifers. Doctoral thesis. Swedish University of Agricultural Sciences. Uppsala. Swedish. 1969.

Findlay, J.K. An update on the roles of inhibin, activin and follistatin as local regulators of folliculogenesos. Biol. Reprod. 48: 15-23. 1993.

Flows, J.A.; Desanti, A.; Tilly, K.I.; Javid, R.O.; Kugu, K. and Johanson, A.L. Vasoactive intestinal peptidemediated suppression of apoptosis in the ovary: potential mechanisms of action and evidence of a conserved anti-atretogenic role through evolution. Endocrinology. 136: 4351-4359. 1995.

Fortune, J.E.; Sirois, J. and Quirk, S.M. The growth and differentiation of ovarian follicles during the bovine estrous cycle. Theriogenology. 29: 95-109. 1988.

Fortune, J. E.. Ovarian follicular growth and development in mammals. Biol. Reprod. 50:225. 1994.

Ginther, O.J.; Bergfelt, D.R.; Kulick, L.J. and Kot, K. Pulsatility of systemic FSH and LH concentration during follicular-wave development in cattle. Theriogenology. 50: 507-519. 1998.

Ginther, O.J.; Wiltbank, M.C.; Fricke, P.M.; Gibbons, J.R. and Kot, K. Selection of the dominant follicle in cattle. Biol. Reprod. 55: 1187-1194. 1996.

Holy, L. Biología de la reproducción bovina. Editorial científico técnica. La Habana. Cuba. Pp: 117-123. 1987.

Hunter, M. Characteristics and cause of the inadequate corpus luteum. J. Reprod. Fert. 43: 91-99. 1991.

Jolly, P.D.; Tisdall, D.J.; Heath, D.A.; Lun, S. and McNatty, K.P. Apoptosis of bovine granulosa cells in relation to steroid synthesis, cyclic adenosine $3^{\prime}, 5^{\prime}$. monophosphate response to follicle stimulating hormone and luteinizing hormone and follicular atresia. Biol. Reprod. 51: 934-944. 1994.

Karsch, F.J.; Bowen, J.M.; Caraty, A.; Evans, N.P. and Moenter, S.M. Gonadotropin-releasing hormone requirements for ovulation. Biol. Reprod. 56: 303-309. 1997.

Kastelic, J.P.; Ko, J.C.H. and Ginther, O.J. Supression of dominant and subordinate ovarian follicles by a proteinaceous fraction of follicular fluid in heifers. Theriogenology. 34: 499-509. 1990.

Knight, P. G., and C. Glister.. Potential local regulatory functions of inhibins, activins and follistatin in the ovary. Reproduction 121:503. 2001.

Knopf, L.; Kastelic, J.P.; Schallember, E. and Ginther, O.J. Ovarian follicular dymanics in heifers. Test of two wave hypothesis by ultrasonically monitoring individual follicles. Domest. Anim. Endocrin. 6: 111 -119. 1989.

Ko, J.C.H.; Kastelic, J.P.; del Campo, M. R. and Ginther, O.J. Effects of a dominant follicle on ovarian follicular dynamics during the oestrous cycle in heifers. J. Reprod. Fert. 91: 511-519. 1991.

Kojima, F. N., and D. J. Patterson. 2003. Guide to Estrus Synchronization of Beef Cattle. University of Missouri, Columbia, MO.

Lussier, J.G.; Matton, P. and Dufour, J.J. Growth rates of follicles in the bovine ovary. J. Reprod. Fertil. 81 : 301-307. 1987.

Monget, P. and Monniaux, D. Growth factors and the control of folliculogenesis. J. Reprod. Fert. Suppl. 49: 321-333. 1995.

Monget $P$, Fabre S, Mulsant P, Lecerf F, Elsen JM, Mazerbourg S, Pisselet C, Monniaux D. Regulation of ovarian folliculogenesis by IGF and BMP system in domestic animals. Domest Anim Endocrinol.;23:139. 2002

Monniaux, D.; Monget, P.; Besnard, N.; Huet, C. and Pisselet, C. Growth factor and antral follicular 
Regulación neuroendocrina del ciclo estral en la hembra bovina Melquisedec Ascanio Pérez

development in domestic rumiants. Theriogenology. 47: 3-12. 1997.

Monniaux, F; Huet, C.; Besnard, N.; Clément, F.; Bosc, H.; Pisselet, C.; Monget, P. and Mariana, J.C. Follicular growth and ovarian dynamics in mammals. J. Reprod. Fert. 112: 25-31. 1997.

Murphy, B.D. y Pescador, N. Control de la foliculogénesis bovina por factores endocrinos y paracrinos. VII Curso Internacional de Reproducción Bovina. Centro Médico Nacional. Siglo XXI. D.F. México. 1997.

Newton, G.R.; Martinod, S.; Hansen, P.J.; Thatcher, W.W.; Siegenthaler, B.; Gerber, C. and Voirol, M.J. Effect of bovine interferon on acute changes in body temperature and serum progesterone concentration in heifers. J. Dairy Sci. 73: 3439-3448. 1990.

Niswender, G.D.; Juengel, J.L. and McGuire, W.J. The strous cycle and early pregnancy. Biol. Reprod. 50: 239-245. 1994.

shea, T.; Hillard, M.A.; Anderson, S.T.; Bindon, B.M.; Findlay, J.K.; Tsonis, C.G. and Wilkens, J.F. Inhibin immunization for increasing ovulatory rate and superovulation. Theriogenology. 41: 3-17. 1994.

Oktay, K.; Schencken, R.S. and Nelson, J.F. Proliferating cell nuclear antigen marks the initiation of follicular growth in the rat. Biol. Reprod. 53: 295-301. 1995.

Peluso, J.J.; Pappalardo, A. and Trolice, M.P. Ncadherin-mediated cell contact inhibits granulosa cell apoptosis in a progesterone dependent manner. Endocrinology. 137: 1196-1203. 1995.

Rajakoski, E. The ovarian follicular system in sexually mature heifers with special reference to seasonal, ciclical and left-right variations. Acta. Endocrinol. 34: 1-6. 1960.

Rivera, C. Regulación neuroendocrina de la función ovárica. En: Transferencia de embriones y biotecnología de la reproducción. Ed. Hemisferio Sur. Argentina. 1993.
Roberts, R. and Smith, M. Luteal function after intrauterine infusion of recombinante bovine interferon. J. Reprod. Fert. 94: 319-325. 1992.

Roche, J.f. and Boland, M.P. Turnover of dominant follicles in cattle of different reproductive states. Theriogenology. 35: 81-90. 1991.

Roche, J.F. Control and regulation of folliculogenesis. A symposium in perspective. Rev. Reprod. 1: 19-27. 1996.

Savio, J.D.; Keenal, L.; Boland, M.P. and Roche, J.F. Ovarian follicular development during the oestrous cycle in heifers. J. Reprod. Fert. 77: 124-130. 1987.

Savio, J.D.; Keenan, L.; Bonald, M.P. and Roche, J.F. Patterns of growth of dominant folicles during the oestrous cycle of heifers. J. Reprod. Fert. 83: 663-671. 1988.

Savio, J.D.; Thatcher, W.W.; Badinga, L.; de la Sota, R.L. and Wolfenson, D. Regulation of dominant follicle turnover during the oestrous cycle in cows. J. Reprod. Fertility. 97: 197-203. 1993.

Silvia, W. and Taylor, M. Relationship between uterine secretion of prostaglandinF 2 alpha induced by oxytocin and endogenous concentrations of $\mathrm{E}_{2}$ y $\mathrm{P}_{4}$ and three stage of the bovine estrous cycle. J. Anim. Sci. 67: 2347-2353. 1989.

Silvia, W.; Lewis, G. and Wilson, J. Hormonal regulation of uterine secretion of prostaglandine $\mathrm{F}_{2}$ alpha. Biol. Reprod. 45: 655-663. 1991.

Sirois, J. and Fortune, J.E. Ovarian follicular dynamics during the estrous cycle in heifers monitored by realtime ultrasonography. Biol. Reprod. 39: 308-317. 1988.

L.J Spicer, CC Chase Jr, L M Rutter. Relationship between serum insulin-like growth factor-l and genotype during the postpartum interval in beef cows. Journal of Animal Science. 80:716. 2002.

Sugino, K.; Norosawa, N.; Nakamura, T.; Takio, K.; Shimasaki, S.; Ling, N.; Titani, K. and Sugino, H. 
Regulación neuroendocrina del ciclo estral en la hembra bovina Melquisedec Ascanio Pérez

Molecular heterogeneity of follistatin an activin-binding protein. J. Biol. Chem. 286: 15579-15587. 1993.

Sunderland, S.J.; Growe, M.A.; Boland, M.P.; Roche, J.F. and Ireland, J.J. Selection, dominance and atresia of follicles during the oestrous cycle of heifers. J. Reprod. Fert. 101: 547-555. 1994.

Tilly, J.L.; Kowalski, K.I.; Johnson, A.L. and Hsuch, A.J.W. Involvement of apoptosis in ovarian follicular atresia and postovulatory regresion. Endocrinology. 129: 2799-2801. 1991.

Wiltbank, M.C.; Shiao, T.F. and Bergfelt, D.R. Prostaglandin $\mathrm{F}_{2} \mu$ receptors in the early bovine corpus luteum. Biol. Reprod. 52: 74-80. 1995.

Zarco, L. Infertilidad debida a asincronia maternoembrionaria en rumiantes. VI Curso Internacional de Reproducción Bovina. Centro Médico Nacional. Siglo XXI. D.F. México. 1995.

Zollers, W.G.; Garverick, H.A.; Smith, M.F.; Moffatt, R.J.; Salfen, B.E. and Youngquist, R.S. Concentration of progesterone and oxitocin receptors in endometrium of postpartum cows expected to have a short or normal oestrus cycle. J. Reprod. Fertility. 97: 329-337. 1993. 